\title{
The Direct Spectroscopy of Positronium Hyperfine Structure Using a Sub-THz Gyrotron
}

\author{
Akira Miyazaki • Takayuki Yamazaki • Taikan Suehara • \\ Toshio Namba • Shoji Asai · Tomio Kobayashi · Haruo Saito • \\ Toshitaka Idehara · Isamu Ogawa · Yoshinori Tatematsu
}

Received: 24 April 2013 / Accepted: 3 July 2013 /

Published online: 25 July 2013

(C) Springer Science+Business Media New York 2013

\begin{abstract}
Positronium is an ideal system for research on Quantum Electrodynamics (QED), especially in a bound state. A discrepancy of 3.9 standard deviations has been found between the measured hyperfine structure (Ps-HFS) and the QED predictions. This may be due to the contribution of unknown new physics or common systematic effects in previous measurements, in all of which the Zeeman effect was used. We propose a new method to directly measure the Ps-HFS using a high power gyrotron. We compare two resonators which have been developed to supply sufficient power to drive the direct transition, a Fabry-Pérot resonator and a ring resonator with a diffraction grating. We plan to perform first direct measurement of Ps-HFS within the next six months.
\end{abstract}

Keywords Positronium · Hyperfine splitting · Gyrotron · Fabry-Pérot resonator · Ring resonator $\cdot$ Diffraction grating

\footnotetext{
A. Miyazaki $(\bowtie) \cdot$ T. Yamazaki $\cdot$ T. Suehara - T. Namba $\cdot$ S. Asai · T. Kobayashi Department of Physics, Graduate School of Science, and International Center for Elementary Particle Physics, The University of Tokyo, Hongo, Bunkyo-ku, Tokyo 113-0033, Japan e-mail: miyazaki@icepp.s.u-tokyo.ac.jp

T. Suehara

Department of Physics, Graduate School of Science, Tohoku University, 6-3 Aoba, Aramaki, Aoba-ku, Sendai, Miyagi 980-8578, Japan

H. Saito

Graduate School of Arts and Sciences, The University of Tokyo, 3-8-1 Komaba, Meguro-ku, Tokyo 153-8902, Japan

T. Idehara $\cdot$ I. Ogawa $\cdot$ Y. Tatematsu Research Center for Development of Far-Infrared Region, University of Fukui, Bunkyo 3-9-1, Fukui 910-8507, Japan
} 


\section{Introduction}

Positronium (Ps), the bound state of an electron and a positron, is a purely leptonic system and is a good system with which to precisely study Quantum Electrodynamics (QED) [1]. Positronium has two spin eigenstates: ortho-positronium (o-Ps, $\mathrm{Spin}=1$, lifetime $=142 \mathrm{~ns}$, three-photon decay) and para-positronium ( $p$-Ps, Spin=0, lifetime $=125 \mathrm{ps}$, two-photon decay). The positronium hyperfine structure (Ps-HFS) is the energy difference between ground-state $o$-Ps and $p$-Ps. Ps-HFS is about $203 \mathrm{GHz}(0.84 \mathrm{meV})$, is significantly larger than the hyperfine structure of the hydrogen atom $(1.4 \mathrm{GHz})[2]$.

Precise measurements have been performed in 1970's and 80's, the results of which are shown in Fig. 1. These results are all consistent, and the average of the two most accurate measurements is $203.38865(67) \mathrm{GHz}(3.3 \mathrm{ppm})$ [3], shown by a green band. A calculation of higher order corrections to the bound state was made in 2000 [4]. The QED prediction is 203.3917(6) GHz (2 ppm), shown as a red band in Fig. 1. A discrepancy of $3.04(79) \mathrm{MHz}(15 \mathrm{ppm})$ is found between the measured value and the QED prediction, which is unlikely to be due to a statistical fluctuation (3.9 standard deviations).

In all previous measurements, the Ps-HFS transition is not observed because $203 \mathrm{GHz}$ is too high a frequency to be handled. A static magnetic field is applied to cause Zeeman mixing, and Ps-HFS is extracted from the Zeeman shift [3]. As mentioned in Ref. [5], there are two possible uncertainties in this method. The first is due to a non-uniformity of the static magnetic field, and the second is due to the non-thermalization of positronium in gas, similar to effects seen in lifetime measurements of $o$-Ps [6]. We have previously proposed a new method to overcome these problems [7].

It is very important to re-measure Ps-HFS using a method totally different from previous experiments. Some independent experiments (using quantum interference [8], optical lasers [9], and a precise magnetic field and correcting gas effect [10])

Fig. 1 Historical plot of Ps-HFS values. Points with error bars show the experimental results with references. Green and red bands show the average of the measured values (the most precise and the latest two results), and the theoretical value calculated up-to $O\left(\alpha^{3} \log \alpha^{-1}\right)$, respectively. $\alpha$ is the fine-structure constant
Experimantal average Theory (2000)

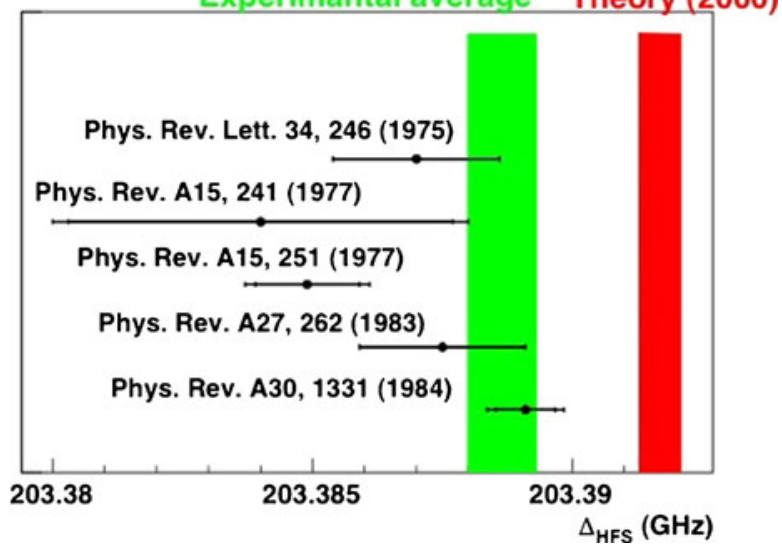


have been performed, but have not yet reached a sufficient level of precision to address the observed discrepancy.

A direct measurement of the Ps-HFS transition without a static magnetic field is free from systematic uncertainties due to magnetic fields. This new method becomes possible by using a gyrotron which can produce high power sub-THz radiation. We are planning to directly measure Ps-HFS with an initial precision of about $0.1 \%$. Although this experiment faces certain technological difficulties connected to the use of sub-THz waves, it is the only method which does not use a static magnetic field, and has great potential to solve the problem in the future.

\section{Direct Measurement of Ps-HFS}

Figure 2 shows a schematic view of the direct transition. $O$-Ps transits directly into $p$-Ps when irradiated by electromagnetic waves of about $203 \mathrm{GHz}$. The transiting $p$ Ps immediately decays into back-to-back two photons (lifetime is $125 \mathrm{ps}$ ), whereas $o$-Ps decays slowly into three photons (lifetime is $142 \mathrm{~ns}$ ). This direct transition can be probed by an increase of two photon decay. High power radiation of over $10 \mathrm{~kW}$ is needed to drive the transition because this is the magnetic dipole transition and severely suppressed (transition rate is $3.37 \times 10^{-8} s^{-1}$ [11]). The radiation frequency should be tuned around $203 \mathrm{GHz} \pm 2 \mathrm{GHz}$ in order to measure the Breit-Wigner resonance.

An optical system composed of a gyrotron and an optical resonator has been developed to produce high power and tunable sub- $\mathrm{THz}$ radiation. Figure 3 a shows a schematic view of the experimental setup. A gyrotron is used as a sub- $\mathrm{THz}$ radiation source, with an output power of about $300 \mathrm{~W}$. An optical resonator accumulates the gyrotron output to obtain a power equivalent to about $20 \mathrm{~kW}$. Positronium is formed inside the resonator and decays into photons ( $\gamma$ rays) which are detected by $\gamma$-ray detectors.

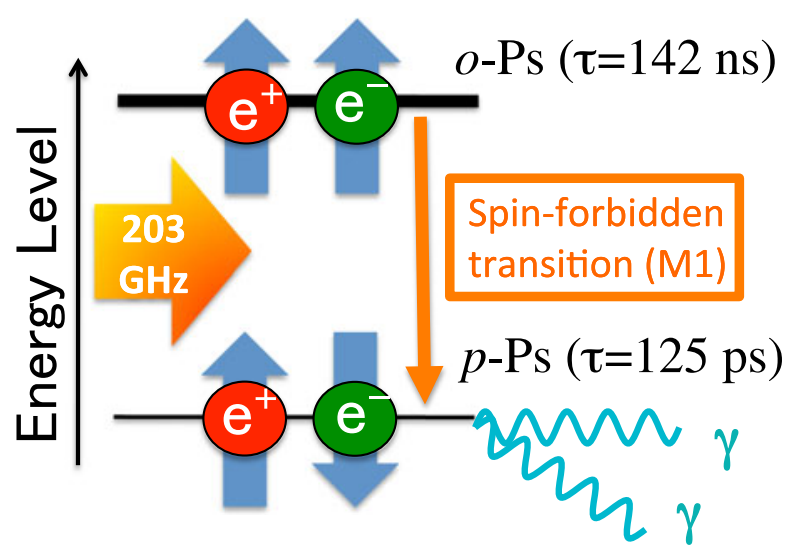

Fig. 2 Schematic diagram of the direct transition of positronium in the ground state 
a

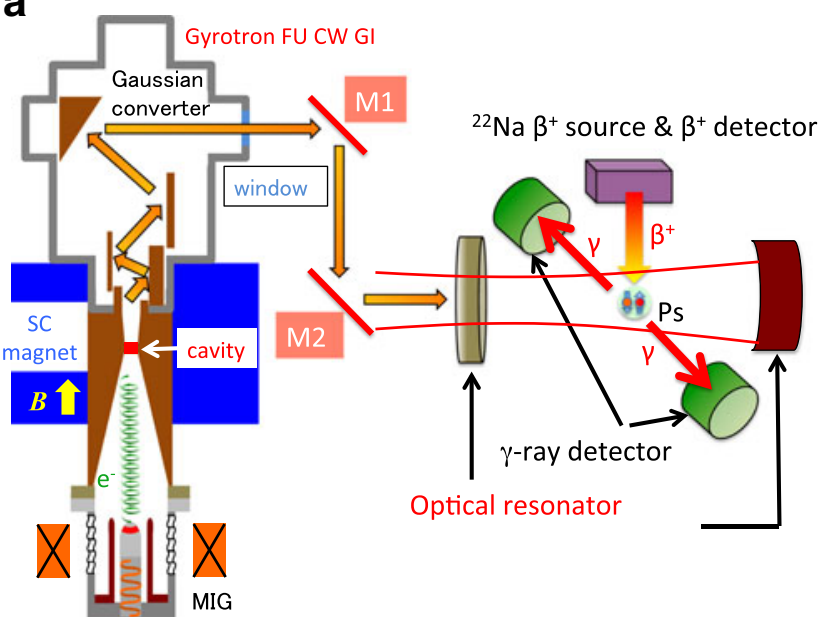

b

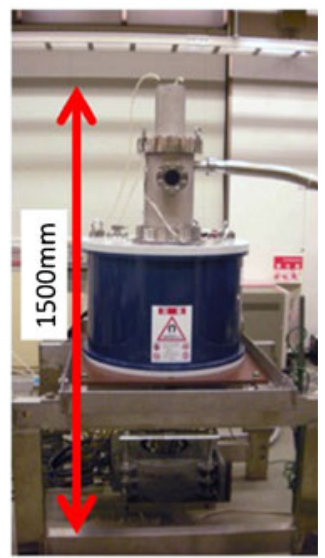

Fig. 3 a Schematic view of the experimental setup. b Photograph of FU CW GI

\subsection{Gyrotron}

Figure 3 b shows a photograph of the gyrotron (FU CW GI) developed specifically for Ps-HFS measurement [12]. The operation mode is the counter rotating $\mathrm{TE}_{52}$ mode, and its output is converted to a Gaussian mode by an internal mode converter as shown in Fig. 4a.

The output power of the Gaussian beam has been measured using a water load. Its peak power is about $300 \mathrm{~W}$ with a condition of duty ratio $30 \%$ and repetition rate $5 \mathrm{~Hz}$. The accelerating voltage and beam current of the magnetron injection gun are $18 \mathrm{kV}$ and $0.5 \mathrm{~A}$, respectively.

a

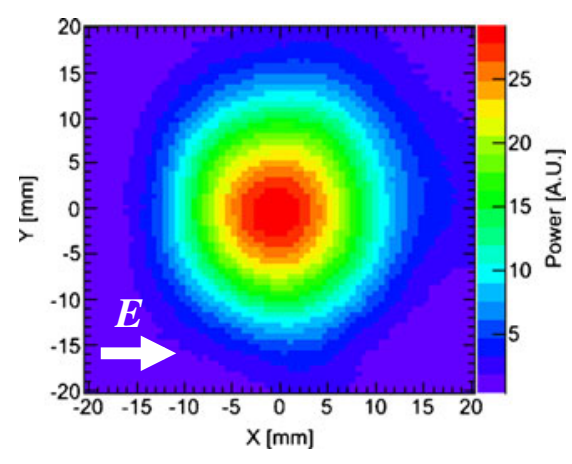

b

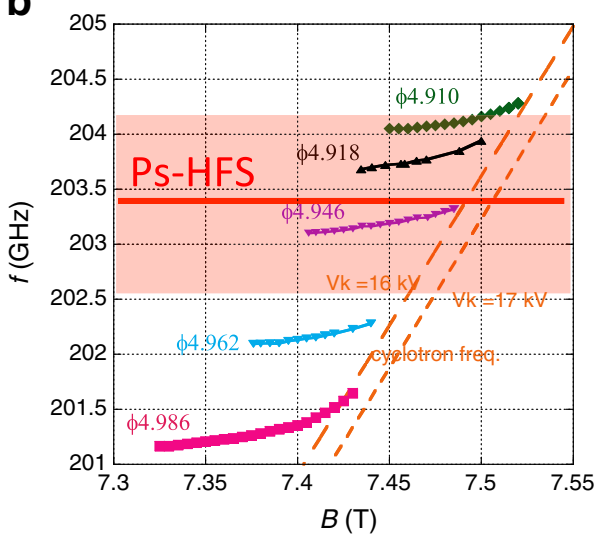

Fig. 4 a Mode pattern measured by an infrared camera after M2 as shown in Fig 3a. b Result of the frequency measurement using a harmonic mixer 
The width of the Ps-HFS Breit-Wigner resonance is about $1.3 \mathrm{GHz}$ (FWHM) corresponding to the lifetime of $p$-Ps. The gyrotron is developed to have interchangeable cavities of different diameters. Figure $4 b$ shows the results of frequency measurement for different cavities. The frequency of the gyrotron is continuously tunable within range of a few hundred $\mathrm{MHz}$ by adjusting the magnetic field strength $B$ according to the formula $f_{c}=e B / 2 \pi m_{0} \gamma$, where $m_{0}$ is the electron rest mass and $\gamma$ is the relativistic factor of the electron. The Ps-HFS value can be measured by selecting appropriate cavities and magnetic field strengths.

\subsection{Optical Resonator}

The Gaussian beam from the gyrotron is accumulated in an optical resonator to drive the direct transition. Two candidates have been developed in parallel: a Fabry-Pérot resonator with a gold mesh, and a ring resonator with a diffraction grating. The direct transition was firstly observed using the Fabry-Pérot resonator [13]. The ring resonator has the advantage of allowing the accumulation of very high power when the gyrotron output increases into the MW range.

\subsubsection{Fabry-Pérot Resonator with a Gold Mesh Mirror}

Figure 5a shows a photograph of the the Fabry-Pérot resonator, which consists of two parallel mirrors. The mirror on the input side, of diameter $50 \mathrm{~mm}$, consists of a gold mesh. It has a high reflectance (over $99 \%$ ) and does not severely disturb the input Gaussian beam. The mirror on the other side is a concave copper mirror (with a radius of curvature of $300 \mathrm{~mm}$ and an expected reflectance of $99.85 \%$ ). A piezoelectric stage under the copper mirror controls the cavity length (about $160 \mathrm{~mm}$ ) with submicron precision. A pyroelectric detector monitors the transmitted radiation through a small hole ( of diameter $0.6 \mathrm{~mm}$ ) on the copper mirror. A second pyroelectric detector outside the resonator monitors the reflected power.

The gold mesh, shown in Fig. 5b, was evaporated onto the high resistivity silicon substrate. The good thermal conductivity $(150 \mathrm{~W} / \mathrm{Km})$ of the silicon enables the mesh to be cooled by water. The mesh parameters (width, gap, and thickness of the mesh and the substrate) was carefully optimized using CST MW studio [14] to avoid interference between the gold mesh and substrate. The S-parameters of the mesh mirror were simulated in the frequency domain solver under periodic boundary conditions.

Two independent parameters, the finesse and coupling, are used to estimate the gain of the optical resonator. The finesse $\mathcal{F}$ is equivalent to the $\mathrm{Q}$ factor and is defined as

$$
\mathcal{F}=\frac{2 \pi}{1-R_{f} R_{e}},
$$

where $R_{f}$ and $R_{e}$ are reflectance of the gold mesh and the copper mirror, respectively [15]. The coupling $C$ is the effective power input into the resonator. It becomes $100 \%$ when all input power enters the resonator. The gain $G$ of the resonator is approximated by $G=C \mathcal{F} / \pi$. 
a

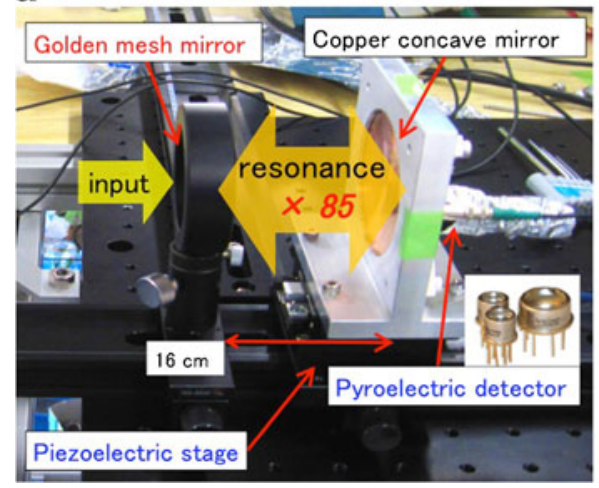

b

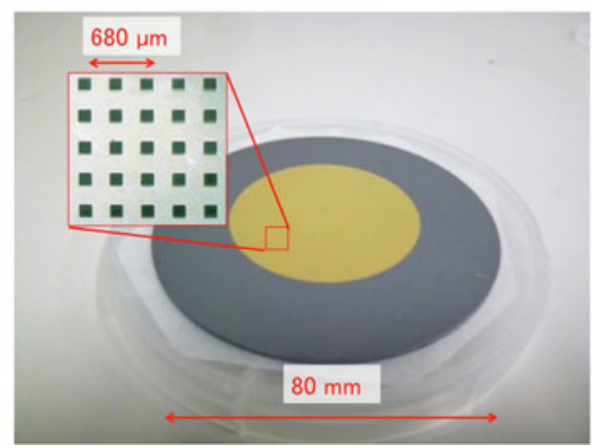

Fig. 5 a Photograph of the Fabry-Pérot resonator. b Gold mesh pattern on a high-resistivity silicon substrate

Figure 6a shows the simulated finesse of the cavity with an optimized mesh mirror. Although the finesse has an inevitable frequency dependence because of the high refractive index of the silicon substrate at $203 \mathrm{GHz}(n=3.45)$, the finesse is over 400 between $201 \mathrm{GHz}$ and $205 \mathrm{GHz}$ and is adequate for the proposed measurement of Ps-HFS. Figure $6 b$ shows the measured resonance of the Fabry-Pérot resonator at $202.1 \mathrm{GHz}$. Experimentally, $\mathcal{F}$ can be obtained by $\mathcal{F}=\frac{\mathrm{FSR}}{\Gamma}$, where FSR is Free Spectral Range $(\lambda / 2=735 \mu \mathrm{m})$, and $\Gamma$ is FWHM of the resonance as shown in Fig. 6b. The measured $\mathcal{F}$ is estimated to be 430 , which is smaller than the simulated value at $202.1 \mathrm{GHz}$ (about 600) due to the finite line width of the gyrotron oscillation $(\Delta f \sim 1 \mathrm{MHz}$ ). The coupling is the fractional decrease of reflected power on

a

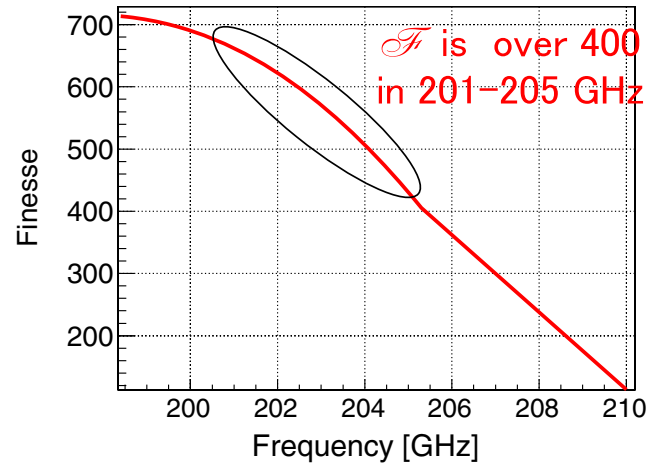

b

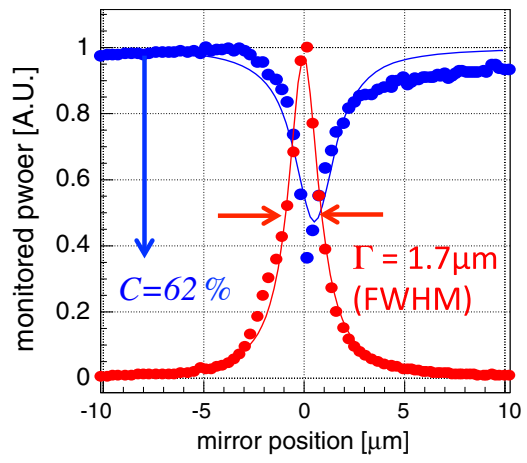

Fig. 6 a Calculated finesse of the Fabry-Pérot resonator with the optimized gold mesh (width=200 $\mu \mathrm{m}$, $\mathrm{gap}=140 \mu \mathrm{m}$, thickness $=1 \mu \mathrm{m}$ ) on the silicon substrate (thickness $=1960 \mu \mathrm{m}$ ). The simulation only includes ohmic losses of the plane wave at the mirrors. b Measured resonance of the new Fabry-Pérot resonator at $202.1 \mathrm{GHz}$. The red points are transmitted power, and the blue points are reflected power. The solid line shows the best fitting Breit-Wigner curves 
resonance, and is measured to be $62 \%$ as shown in Fig. 6b. Therefore, the measured gain of the new Fabry-Pérot resonator is about 85 . The equivalent power inside the resonator is $25 \mathrm{~kW}$ in case of $300 \mathrm{~W}$ input power. The accumulated power is affected by the line width of the gyrotron oscillation, but $25 \mathrm{~kW}$ is sufficient for this measurement.

\subsubsection{Ring Resonator with a Diffraction Grating}

We have also developed a ring cavity with a diffraction grating and compared its performance to the Fabry-Pérot resonator. It consists of three copper mirrors as shown in Fig. 7. The main mirror is a diffraction grating made of copper. It is easier to watercool this grating than the mesh mirror of the Fabry-Pérot resonator. The diffracted beam enters the cavity which resonantes when the length of the triangular optical path is an integer multiple of the wavelength $(\lambda=1.47 \mathrm{~mm})$. The reflected beam ( 0 th diffraction) does not return into the incident direction, an advantage of the ring resonator over the Fabry-Pérot resonator. Denoting the incident angle $\alpha$, diffraction angle $\beta$, and spacing of the grating $d$, the grating equation is given by

$$
d(\sin \alpha+\sin \beta)=m \lambda(m=0, \pm 1, \pm 2, \ldots) .
$$

According to Eq. 2, the spacing $d$ is uniquely determined if the cavity shape is fixed. The grating should be designed to reduce higher order diffractions since they cause loss of input power and therefore reduce the coupling. We chose $\alpha=60^{\circ}, \beta=15^{\circ}$ and $m=-1$, giving a spacing $d$ of $1.31 \mathrm{~mm}$.

Diffraction gratings with grooves of different shapes and depths have been fabricated and compared. Figure 8 shows a typical resonance. Table 1 summarizes the performance of different gratings. Gratings with shallower grooves have a higher finesse, however the coupling decreases if the grooves are too shallow. This is because a small amount of scattered radiation by the grating surface becomes comparable to the diffracted beam power. The circular type grating has cylindrical grooves

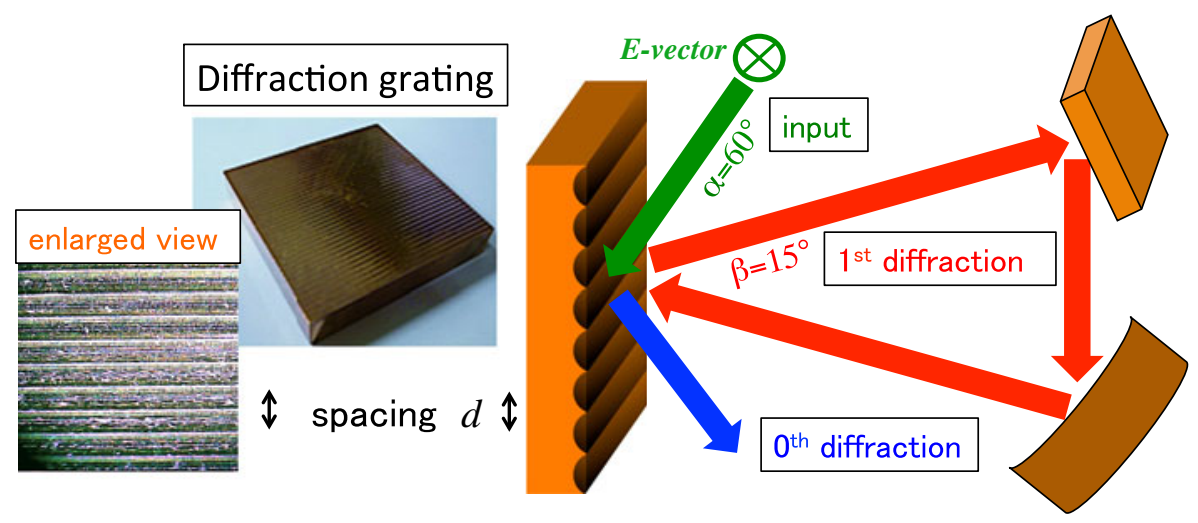

Fig. 7 Photograph of the diffraction grating (left) and schematic view of the ring resonator (right) 
Fig. 8 Obtained resonance of the ring resonator at $202.9 \mathrm{GHz}$ when grating type is circular, and depth is $48 \mu \mathrm{m}$. The definition of the points and lines are the same as that of Fig. $6 \mathrm{~b}$

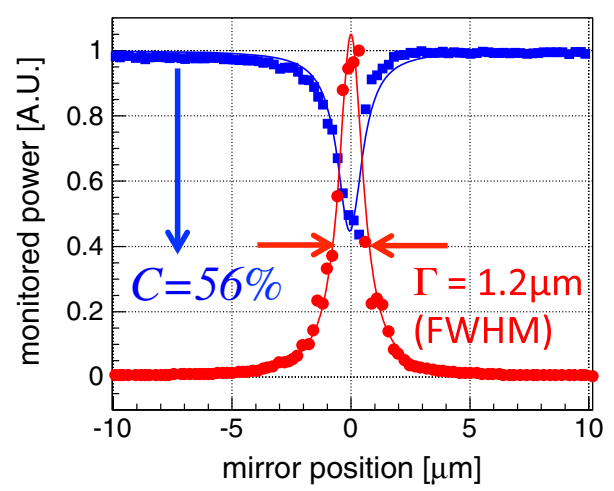

with sharp edges, while the sine type grating grooves have a a smooth sine-wave profile. ${ }^{1}$ The circular shape grating has a higher finesse than a sine shape grating with the same groove depth. The best performance is obtained by the circular type with a groove depth of $48 \mu \mathrm{m}$ as shown in Fig. 8. The gain $G$ is about 110, and accumulated power inside the resonator is expected to be over $30 \mathrm{~kW}$.

The optical performance of the ring resonator is better than that of the Fabry-Pérot resonator. However, the Fabry-Pérot resonator has a significant advantage when we consider Ps formation. A positron source is used to form Ps with an electron in a gas molecule [5]. Since the optical path of the ring cavity is split into two separate paths, positronium cannot be efficiently formed in both. The direct transition signal (i.e. two photons from transiting $p$-Ps) from the ring resonator is half of that from the FabryPérot resonator. We have therefore decided to use the Fabry-Pérot resonator for the first measurement of Ps-HFS.

Although the ring resonator is not suitable for Ps-HFS measurement using a positron source, it will be an excellent solution if a positron beam [16] is introduced in the future. Because of the split optical path, the energy density of the diffraction grating is half as small as that of the Fabry-Pérot resonator, which prevents saturation of the transition probability of positronium. If positronium can be efficiently formed in the large optical-path region by a positron beam, the transition signal from the resonator will be drastically enhanced.

The ring resonator has another possible application. The equivalent power inside the resonator will be of order $100 \mathrm{MW}$ when we introduce a MW-class gyrotron [17]. Such high power millimeter waves can be used to search for hypothetical light particles predicted in particle physics [18]. The diffraction grating can endure a power of $1 \mathrm{MW}$, whereas the gold mesh of the Fabry-Pérot resonator definitely cannot. An additional advantage is that the beam reflected from the resonator does not return to the input source. In contrast, the reflected beam from the Fabry-Pérot resonator inevitably returns into the gyrotron, destabilizing the gyrotron oscillation [19], which should be prevented, especially when a MW-class gyrotron is used.

\footnotetext{
${ }^{1}$ The popular blazed type grating is not suitable because its asymmetrical shape cannot maximize both finesse and coupling in the case of our ring cavity whose optical path is an isosceles triangle.
} 
Table 1 Comparison of different gratings at $202.9 \mathrm{GHz}$. The small coupling of circular type with depth of $36 \mu \mathrm{m}$ may be due to scratches on the surface

\begin{tabular}{llll}
\hline Shape & Depth $(\mu) \mathrm{m}$ & $\mathcal{F}$ & $C$ \\
\hline circular & 72 & 405 & $59 \%$ \\
circular & 48 & 628 & $56 \%$ \\
circular & 36 & 636 & $18 \%$ \\
circular & 24 & 910 & $24 \%$ \\
sine & 36 & 576 & $45 \%$ \\
\hline
\end{tabular}

\section{Current Status and Future Prospect}

We have already observed the direct transition at $202.9 \mathrm{GHz}$ as shown in Fig. 9 (solid point) $[13,20]$. The dotted line in Fig. 9 is the theoretical calculation of the BreitWigner shape of the transition. We will measure the transition at four additional points $(201.8 \mathrm{GHz}, 203.4 \mathrm{GHz}, 204 \mathrm{GHz}$ and $205.3 \mathrm{GHz})$ within half a year. The Ps-HFS will be directly measured with precision of about $0.1 \%$ for the first time.

In order to test the Ps-HFS discrepancy, four technological progresses are needed. First, a precise power detector (relative accuracy is better than $0.3 \%$ ) should be developed in the $200 \mathrm{GHz}$ region. Secondly, the quality and stability of the gyrotron oscillation should be improved. The line width of the oscillation frequency must be stable and less than $100 \mathrm{kHz}$. Thirdly, a high power gyrotron with continuously tunable frequency should be developed. A reflective gyro-backward-wave oscillator has been theoretically proposed, but not yet put into practice [21]. Lastly, a positron beam should be used to obtain better statistics.

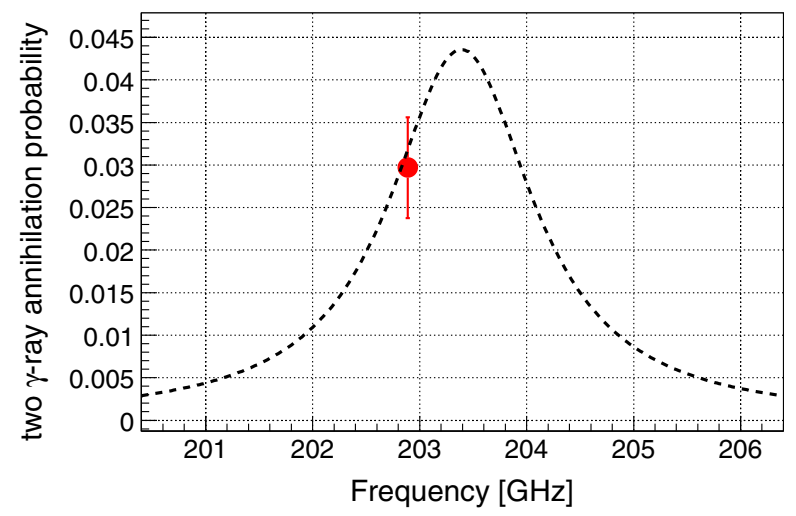

Fig. 9 Transition curve of the Ps-HFS transition. The solid point shows the measured transition (only statistical error is shown); the dotted line does a theoretical prediction 


\section{Summary}

There is a discrepancy between the measured Ps-HFS values and QED predictions. We propose that the direct measurement of Ps-HFS is free from the possible systematic uncertainties due to the magnetic field present in all the previous measurements. A gyrotron with an internal mode converter has been developed, whose frequency can be tuned to scan the entire resonance curve of the Ps-HFS transition. In order to obtain the $20 \mathrm{~kW}$ equivalent power needed to efficiently drive the direct transition, a Fabry-Pérot resonator and a ring resonator were developed and compared. We will directly measure the Ps-HFS using the Fabry-Pérot resonator for the first time within half a year.

Acknowledgments Sincere gratitude is extended to Dr. Daniel Jeans for the useful discussions.

\section{References}

1. T. Namba, Prog. Theor. Exp. Phys., 04D003 (2012).

2. N.F. Ramsey, Rev. Mod. Phys. 62, 541 (1990).

3. A.P. Mills, Phys. Rev. A 27, 262 (1983); M.W. Ritter et.al., Phys. Rev. A 30, 1331 (1984).

4. K. Melnikov, et al., Phys. Rev. Lett. 86, 1498 (2001); B.A. Kniehl and A.A. Penin Phys. Rev. Lett. 85, 5094 (2000); R.J. Hill, Phys. Rev. Lett. 86, 3280 (2001).

5. S. Asai, et al., J Infrared Milli Terahz Waves 33, 766 (2012).

6. S. Asai et al., Phys. Lett. B 357, 475 (1995); O. Jinnouchi et al., Phys. Lett. B 572, 117 (2003); Y. Kataoka et al., Phys. Lett. B 671, 219 (2009).

7. S. Asai et al., AIP Conf. Proc. 1037, 43 (2008) and arXiv:0805.4672.

8. V.G. Baryshevsky et al., J. Phys. B 22, 2835 (1989); V.G. Baryshevsky et al., Phys. Lett. A 136, 428 (1989); S. Fan et al., Phys. Lett. A 216, 129 (1996); Y. Sasaki and A. Miyazaki et al., Phys. Lett. B 697, 121 (2011).

9. D.B. Cassidy et al., Phys. Rev. Lett. 109, 073401 (2012).

10. A. Ishida et al., Hyperfine Interact 212, 133 (2012).

11. V.V. Burdyuzha et al., Astro. Lett. 25, 3 (1999).

12. Y. Tatematsu et al., J. Infrared Milli Terahz Waves, 33, 292 (2012).

13. T. Yamazaki and A. Miyazaki et al., Phys. Rev. Lett. 108, 253401 (2012).

14. CST Microwave Studio 2011, CST, Computer Simulation Technology AG, http://www.cst.com.

15. E. Hecht, OPTICS, Addison-Wesley Publishing Company, international edition, fourth edition, p 423, 2002.

16. K. Wada et al., Eur. Phys. J. D 66, 37 (2012).

17. K. Sakamoto et al., Nucl. Fusion 49, 095019 (2009).

18. J. Jaeckel, Andreas Ringwald, arXiv:1002.0329.

19. T.M. Antonsen et al., Phys. Fluids. B 4, 12, 4131 (1992).

20. T. Idehara et al., J. Infrared Milli Terahz Waves, 33, 667, (2012).

21. T.H. Chang and T. Idehara et al., J. Appl. Phys. 105, 063304 (2009). 